where $A=\left(\overline{g_{n} / r^{3}}\right) \mu_{N}{ }^{2} / 10, \mu_{1}=\left(1+g_{p}-g_{n}\right) \mu_{N} / 2, \mu_{2}=\left(1-g_{p}+g_{n}\right) \mu_{N} / 2$, and $\mu_{3}=\left(g_{p}+g_{n}\right) \mu_{N} / 2$. The perturbation term of the energy we write $\epsilon \mp g_{\mu_{N}} H$, where $g$ is the ratio of magnetic to mechanical moment of the system in the state of which the energy for $H=0$ is $\epsilon$, in the usual perturbation procedure (setting $g_{p}=-5, g_{n}=-1.1, g_{p} A=a$ ):

$$
\left|\begin{array}{ccc}
-2 a \mp \mu_{1} H-\left(\epsilon \mp g \mu_{N} H\right) & 6 a & -3 a / \sqrt{ } 2 \\
6 a & 4 a \mp \mu_{\mu_{2} H-\left(\epsilon \mp g \mu_{N} H\right)} & 3 a / \sqrt{ } 2 \\
-3 a / \sqrt{ } 2 & 3 a / \sqrt{ } 2 & 2 a \mp \mu_{3} H-\left(\epsilon \mp g \mu_{N} H\right)
\end{array}\right|=0 .
$$

The lowest root for $H=0$ is $\epsilon / a=-6.69$, which, inserted in the determinant, leaves only the terms in $H$ (we neglect Paschen-Back terms in $H^{2}$ ), leading to

$$
g=\frac{\frac{\mu_{1}}{\mu_{N}}\left|\begin{array}{cc}
4-\epsilon / a & 3 / \sqrt{ } 2 \\
3 / \sqrt{ } 2 & 2-\epsilon / a
\end{array}\right|+\frac{\mu_{2}}{\mu_{N}}\left|\begin{array}{cc}
-2-\epsilon / a & -3 / \sqrt{ } 2 \\
-3 / \sqrt{ } 2 & 2-\epsilon / a
\end{array}\right|+\frac{\mu_{3}}{\mu_{N}}\left|\begin{array}{cc}
-2-\epsilon / a & 6 \\
6 & 4-\epsilon / a
\end{array}\right|}{\left|\begin{array}{cc}
4-\epsilon / a & 3 / \sqrt{ } 2 \\
3 / \sqrt{ } 2 & 2-\epsilon / a
\end{array}\right|+\left|\begin{array}{cc}
-2-\epsilon / a & -3 / \sqrt{ } 2 \\
-3 / \sqrt{ } 2 & 2-\epsilon / a
\end{array}\right|+\left|\begin{array}{cc}
-2-\epsilon / a & 6 \\
6 & 4-\epsilon / a
\end{array}\right|}=-0.60, \text { whereas }\left|g_{\exp }\right|=0.7_{5} .
$$

\title{
Erratum: Thermal Expansion and the Ferromagnetic Change in Volume of Nickel
}

Clarke Williams, Columbia University

(Phys. Rev. 46, 1011, 1934)

THE coordinates of the point on Fig. 5, referred to in the third paragraph

of the first column on page 1014 , are Temperature $450^{\circ} \mathrm{C}$, and Coef. of Exp. $15.9 \times 10^{-6}$. This point was shown in Fig. 5 in the proof but disappeared subsequent to the return of the proof to the printer. 